\title{
Impact of Mineral Precipitation on Flow and Mixing in Porous Media Determined by Microcomputed Tomography and MRI
}

\section{Authors: Joshua A. Bray, Ellen G. Lauchnor, George D. Redden, Robin Gerlach, Yoshiko Fujita, Sarah L. Codd, \& Joseph D. Seymour}

This document is the unedited author's version of a Submitted Work that was subsequently accepted for publication in Environmental Science \& Technology, copyright @ American Chemical Society after peer review. To access the final edited and published work, see https:// doi.org/10.1021/acs.est.6b02999.

Bray JM, Lauchnor EG, Redden GD, Gerlach R, Fujita Y, Codd SL, Seymour JD, "Impact of Mineral Precipitation on Flow and Mixing in Porous Media Determined by Microcomputed Tomography and MRI," Environmental Science \& Technology, 2017 February 7; 51 (3), 1562-1569. doi: $10.1021 / a c s . e s t .6 b 02999$ 


\title{
Impact of Mineral Precipitation on Flow and Mixing in Porous Media Determined by Microcomputed Tomography and MRI
}

\author{
Joshua M. Bray, Ellen G. Lauchnor,George D. Redden,Robin Gerlach,Yoshiko Fujita, \\ Sarah L. Codd, and Joseph D. Seymour
}

Precipitation reactions influence transport properties in porous media and can be coupled to advective and dispersive transport. For example, in subsurface environ-ments, mixing of groundwater and injected solutions can induce mineral supersaturation of constituents and drive precipitation reactions. Magnetic resonance imaging (MRI) and microcomputed tomography ( $\mu$-CT) were employed as complementary techniques to evaluate advection, dispersion, and formation of precipitate in a 3D porous media flow cell. Two parallel fluids were flowed concentrically through packed glass beads under two relative flow rates with $\mathrm{Na}_{2} \mathrm{CO}_{3}$ and $\mathrm{CaCl}_{2}$ in the inner and outer fluids, respectively.

$\mathrm{CaCO}_{3}$ became supersaturated and formed a precipitate at the mixing interface between the two solutions. Spatial maps of changing

\begin{abstract}
local velocity fields and dispersion in the flow cell were generated from MRI, while high resolution $\mu$-CT imaging visualized the precipitate formed in the porous media. Formation of a precipitate minimized dispersive and advective transport between the two fluids and the shape of the precipitation front was influenced by the relative flow rates. This work demonstrates that the combined use of MRI and $\mu-C T$ can be highly complementary in the study of reactive transport processes in porous media.
\end{abstract}

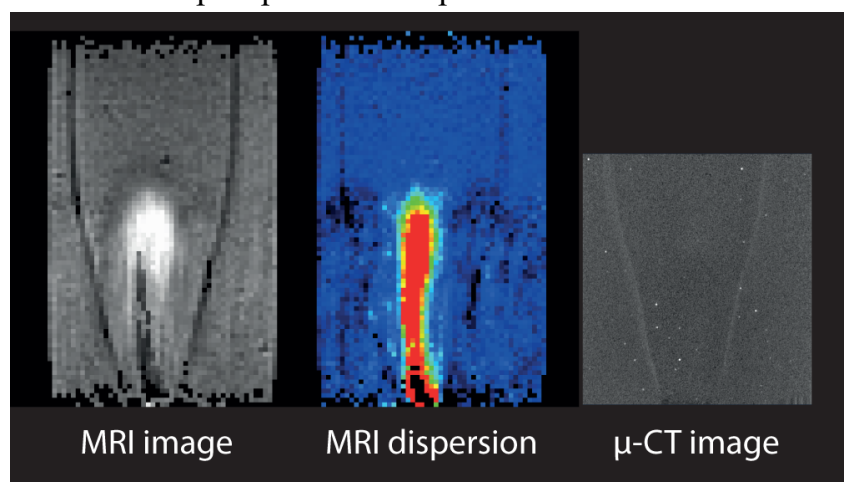

The interplay between precipitation/dissolution reactions and transport processes in porous media has long been studied for industrial reactor design ${ }^{1}$ and has relevance for subsurface processes. ${ }^{2-4}$ Formation of precipitates (by design, uninten-tionally, or in natural systems) can exert control on porous media permeability, flow paths, solute mixing, and volume-averaged reaction rates. 5,6 When precipitation or dissolution reactions occur in porous media, properties such as porosity and permeability evolve with the extent of reaction, creating dynamic feedback between transport and reactive processes. ${ }^{7}$ Predicting and/or controlling mineral precipitation and dissolution in the subsurface is currently a topic of significant interest for the application of engineered calcium carbonate $\left(\mathrm{CaCO}_{3}\right)$ precipitation in the context of enhancing the geological storage of $\mathrm{CO}_{2}$, enhanced oil recovery, soil stabilization, and environmental remediation. ${ }^{8}$ In coastal regions, mixing of fresh and saline groundwater can also influence carbonate chemistry and drive precipitation or dissolution of $\mathrm{CaCO}_{3} \cdot{ }^{9}$ Understanding the relative influence of diffusion, advection, and dispersion on mixing and precipitation reactions in the

subsurface is a formidable challenge. ${ }^{10}$ This manuscript describes a set of experiments in which X-ray microcomputed tomography ( $\mu-\mathrm{CT}$ ) imaging and magnetic resonance imaging (MRI) techniques are used to observe $\mathrm{CaCO}_{3}$ mineral formation in a porous media column and investigate the influence on transport dynamics.

The dependence of $\mathrm{CaCO}_{3}$ dissolution and precipitation in porous media on chemical parameters such as $\mathrm{pH}$, temperature, and salinity has been well-characterized. 9 Flow experiments have focused largely on $2 \mathrm{D}$ systems in microchannels $5,11,12$ and porous media in $2 \mathrm{D}$ cells ${ }^{12-14}$ using optical dye tracer methods. Extending flow studies to 3D coaxial mixing geometries is important because it eliminates edge effects resulting from the interaction of the fluid and precipitates with reactor walls. The inherent challenge is that this requires nondestructive visual-ization techniques capable of imaging morphology and hydrodynamics within opaque, porous flow systems. MRI and 


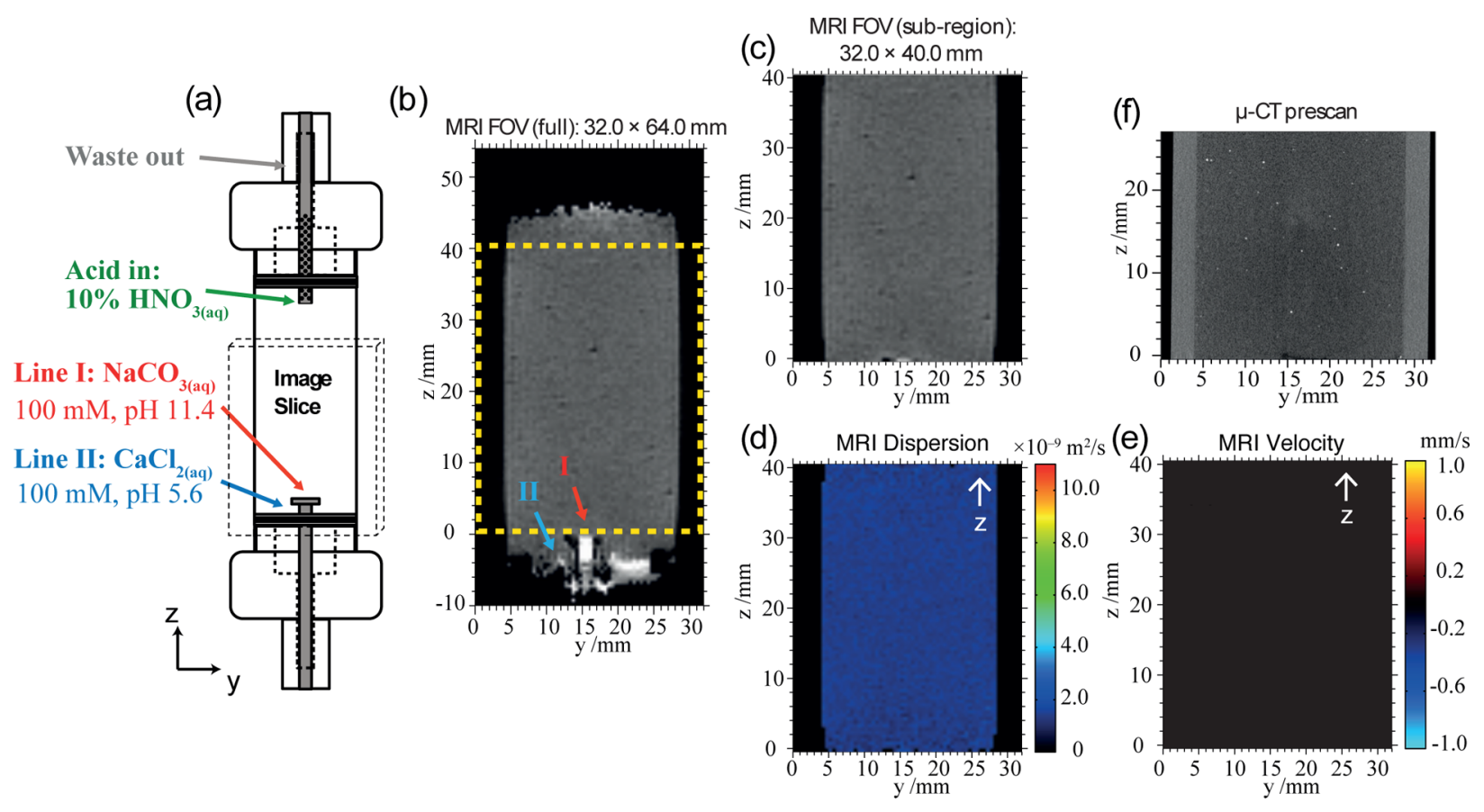

Figure 1. (a) Flow cell schematic diagram showing the concentric inner (Line I) and outer (Line II) inlets, the waste outlet, and the acid injection line. The dashed box shows the MRI image slice, which has an in-plane resolution of $500 \times 500 \mu \mathrm{m}^{2}$ and $1000 \mu \mathrm{m}$ thickness. The MRI ${ }^{1} \mathrm{H}$ signal intensity image (in arbitrary units) is shown for (b) the full field-of-view (FOV) and (c) the subregion used for analysis. Maps of (d) effective diffusion and (e) velocity determined from PGSE-MRI data for "no-flow" conditions with motion sensitization in the $z$-direction. (f) $\mu$-CT scan of clean beadpack prior to precipitation.

$\mu$-CT are two 3D techniques that provide complementary information via their respective contrast types.

The use of MRI for characterizing fluids in porous media is well-established. ${ }^{15}$ The MRI signal originates from the magnetic moments of nuclear spins, and most imaging is done using hydrogen nuclei $\left({ }^{1} \mathrm{H}\right)$ in water. Image intensity depends primarily on local spin density (i.e., water content) but may additionally depend on the $T_{1} / T_{2}$ signal relaxation times via image timing parameters. Correction of relaxation effects can produce quantitative spin density mapping, but simple changes in local water content may be visualized qualitatively via changes in image intensity. In the context of flowing aqueous solutions, the $\mathrm{H}_{2} \mathrm{O}$ molecules serve as endogenous tracers. Additionally, pulsed magnetic field gradients may be used to detect both advective and stochastic components of fluid displacement in porous media. Thus, data may be analyzed to determine the volume-averaged fluid velocity and coefficient of dispersion $\left(D^{*}\right)$, and both quantities can be spatially mapped in heterogeneous samples. ${ }^{16}$ This information can be used in porous media studies to evaluate changes in porosity and preferential flow paths caused by mineral precipitation or dissolution. MRI has been applied to characterize transport changes in $3 \mathrm{D}$ porous media experiments due to biologically induced precipitation. ${ }^{17,18}$ Changes in MRI signal intensity have been used to detect changes in the local water content, such as by pore occlusion during ureolytically driven calcite precipitation. $^{19}$

$\mathrm{X}$-ray computed tomography (CT) images are generated by mapping differences in X-ray attenuation of materials in a sample. ${ }^{20} \mathrm{X}$-ray CT has been applied to analyzing properties and processes in porous media, including pore space geometry, mineralization, and dissolution reactions. ${ }^{4,21,22}$ With an instrument capable of X-ray $\mu$-CT imaging, spatial resolution of pore structure down to $\sim 10 \mu \mathrm{m}$ is achievable-generally a higher spatial resolution than possible with MRI, which is routinely on the order of $\sim 100 \mu \mathrm{m}$ for a comparable measurement time period and sample size.

Using $\mu$-CT, the spatial distribution of $\mathrm{CaCO}_{3}$ precipitates formed in a porous medium can be determined and correlated with permeability and transport dynamics observed by MRI during flow. To our knowledge, this combination of MRI and $\mu$-CT is a novel approach for noninvasively characterizing the coupling between transport and reaction in porous media. The two techniques have previously been combined to study flow in compressed foams, with MRI used for velocimetry and $\mu$-CT structural images used to inform lattice-Boltzman simulations. ${ }^{23}$ Very few environmental studies have taken advantage of these complementary techniques. Two studies on ponded infiltration in soils combined MRI to monitor water content during infiltration and drainage with X-ray CT to provide 3D maps of the soil pore structure, ${ }^{24,25}$ and Rigby et al. also combined the two techniques to investigate mercury intrusion into nanoporous silica materials during porosimetry. ${ }^{26}$ However, these studies primarily focused on using MRI to spatially image water content. The work presented here combines these methods to investigate dynamics of precipitation due to mixing of two fluids in porous media by spatially mapping dispersion and velocity using MRI and imaging precipitate formation using $\mu$ CT.

We investigated the effect of modifying reactant flow rates on locus and structure of $\mathrm{CaCO}_{3}$ precipitate deposition during adjacent flow of $\mathrm{CaCl}_{2}$ and $\mathrm{Na}_{2} \mathrm{CO}_{3}$ solutions in a model 3D porous medium. A flow cell with annular reactant injection was used to create solution-solution mixing interfaces under parallel flow in a porous medium, while preventing contact of the mixing/reaction zone with the cell walls. Our results supported the manipulation of flow rates to control precipitate deposition 
Table 1. Reynolds and Péclet Numbers and Fluid Velocities for the Two Fluid Flow Ratios, Determined at the Inlet Positions and in the Region Where Inner and Outer Velocities Have Equilibrated (beyond the Entrance Length) for Both Flow Ratios

\begin{tabular}{|c|c|c|c|c|c|c|c|c|c|}
\hline \multirow[b]{2}{*}{ flow ratio (outer:inner) } & \multicolumn{3}{|c|}{ outer inlet } & \multicolumn{3}{|c|}{ inner inlet } & \multicolumn{3}{|c|}{ equilibrated region } \\
\hline & $R e$ & $\mathrm{Pe}$ & velocity/ $\left(\mathrm{mm} \mathrm{s}^{-1}\right)$ & $\operatorname{Re}$ & $\mathrm{Pe}$ & velocity $/\left(\mathrm{mm} \mathrm{s}^{-1}\right)$ & $\operatorname{Re}$ & $\mathrm{Pe}$ & velocity $/\left(\mathrm{mm} \mathrm{s}^{-1}\right)$ \\
\hline $1: 1$ & 0.04 & 15 & 0.11 & 7.3 & 3153 & 22.5 & 0.07 & 31 & 0.22 \\
\hline $3: 1$ & 0.05 & 23 & 0.17 & 3.7 & 1584 & 11.3 & 0.07 & 31 & 0.22 \\
\hline
\end{tabular}

and demonstrated the utility of the combined use of X-ray CT and MRI to study reactive transport in porous media.

\section{METHODS}

Flow Cell Construction. A flow cell was constructed using a glass HPLC column (Omnifit Benchmark, $25 \mathrm{~mm}$ i.d.) modified to have separate, coaxial inlets for an inner flow of $\mathrm{Na}_{2} \mathrm{CO}_{3}$ (100 mM, pH 11.4) and outer flow of $\mathrm{CaCl}_{2}$ (100 $\mathrm{mM}, \mathrm{pH}$ 5.6) (Figure 1a). The measured solution densities for $\mathrm{CaCl}_{2}$ and $\mathrm{Na}_{2} \mathrm{CO}_{3}$ were 1.004 and $1.007 \mathrm{~g} / \mathrm{cm}^{3}$, respectively. The cell was wet-packed using borosilicate glass microspheres (180-212 $\mu \mathrm{m}$, Cospheric Inc.) in deionized water, giving a calculated initial porosity of $\phi=0.4$ for random, close-packed beads. ${ }^{27}$ Mesh screens on the inlets secured beads within the cell. Flow was vertically upward, and the outlet at the top of the cell included an outer line for the mixed waste fluid and an inner port for injection of $10 \% \mathrm{HNO}_{3}$ acid to prevent clogging of the waste line.

Magnetic Resonance Imaging (MRI). MRI was performed on a Bruker Super Wide Bore imaging system using a $37 \mathrm{~mm}$ i.d. probe with a ${ }^{1} \mathrm{H}$ frequency of $300 \mathrm{MHz}$. Images were encoded at $500 \times 500 \mu \mathrm{m}^{2}$ resolution in a $1000 \mu \mathrm{m}$ slice through the center of the flow cell as shown in Figure 1a using a spin-echo imaging sequence with $16.42 \mathrm{~ms}$ echo time, $2.0 \mathrm{~s}$ repetition time, read/phase/slice gradients of 0.018/0.19/0.073 $\mathrm{T} / \mathrm{m}$, a readout of duration $2.560 \mathrm{~ms}$ and $50 \mathrm{kHz}$ acquisition bandwidth, and gradient stabilization delays of $195 \mu$ s (using active gradient compensation). The $90^{\circ}$ excitation was a $2.0 \mathrm{~ms}$ sinc3 radiofrequency (RF) pulse, and power was adjusted by $0.3 \mathrm{~dB}$ when switching from pure water to the reactants to compensate for increased power absorption due to the increased conductivity. Fluid displacement encoding was achieved using a standard 8-step pulsed-gradient spin-echo (PGSE) preparation ${ }^{15}$ with magnetic field gradients of duration $\delta=2 \mathrm{~ms}$, separation $\Delta=10 \mathrm{~ms}$, and amplitude $g_{\max }=315 \mathrm{mT} /$ $\mathrm{m}$. The sequence was repeated, alternating between in-plane motion encoding in the longitudinal direction $(+z$, along the direction of flow) and the transverse direction $( \pm y)$, with 34 min required for each scan.

${ }^{1} \mathrm{H}$ signal intensity maps were obtained without correcting for relaxation effects by using the zero-gradient $(g=0 \mathrm{mT} / \mathrm{m})$ PGSE image data. This approach suffices to show qualitative changes in the local water content. The RF transceiver efficiency is uniform near the center of the hardware, but signal is attenuated toward the edges. Therefore, spatial variation in the detector sensitivity was determined using an image acquired under no-flow conditions, and this was used for posthoc correction of the apparent signal nonuniformity. The nonuniformity correction assumes that the initial packing is uniform; this was validated using $\mu$-CT, which is not subject to the same artifacts as MRI. Additionally, bright regions may occur near fluid inlets due to an "in-flow artifact". Fluid that has been subjected to multiple radiofrequency (RF) pulses within the cell reaches a steady-state (saturated) magnetization, but freshly injected fluid has its full, equilibrium magnetization and appears brighter, artificially giving the appearance of higher fluid content in those voxels. A subregion of the total image was analyzed for determination of velocity and hydrodynamic dispersion (Figure 1b), where the field-of-view extends from just past the inner reactant inlet $(z=0 \mathrm{~mm})$ to a point $40 \mathrm{~mm}$ downstream (located $\sim 20 \mathrm{~mm}$ before the outlet). The dispersion and velocity maps were calculated using Prospa (Magritek Inc.) software, which determines $D^{*}$ using leastsquares fitting to signal attenuation and $v$ from the complex image phase in each voxel. These calculations are inherently insensitive to variation in detector sensitivity, and therefore no spatial correction was applied. For more information on MRI theory and methods, see the Supporting Information.

X-ray Microcomputed Tomography $(\mu-\mathrm{CT})$. X-ray $\mu$-CT images of the porous medium and precipitate were taken under no-flow conditions before and after the flow experiments. A SkyScan $1173 \mu$-CT system (Bruker-microCT, Belgium) was used to obtain sets of X-ray projection images of the flow cell as it was rotated around the vertical axis as oriented in Figure 1a. Scans were performed at an X-ray production voltage of $130 \mathrm{kV}$ and current of $61 \mu \mathrm{A}$ with a $0.25 \mathrm{~mm}$ brass filter. The scan resolution corresponded to a pixel size of $15.3 \mu \mathrm{m}$, and the exposure time was $2 \mathrm{~s}$ per scan with a rotational step of $0.7^{\circ}$.

Reconstruction of $\mu$-CT images was performed using SkyScan NRecon software (Bruker-microCT), which uses the Feldkamp algorithm ${ }^{28}$ to generate a series of horizontal crosssection images with a resolution of $15.3 \mu \mathrm{m}$, which was also the depth of each cross-section in the series. Horizontal cross sections at $30 \mathrm{~mm}$ from the inlet were analyzed to determine the thickness of the precipitate region and the area for flow of the reacting fluids. The analysis was performed in ImageJ, ${ }^{29}$ where images were segmented into black and white based on pixel intensity (brightness) using the Otsu method for automatic threshold selection. ${ }^{30}$ In the segmented images, white indicated higher density regions, namely the precipitate zone, and black indicated less dense regions. The ImageJ particle analysis function was used to identify the inner and outer boundaries of the precipitate region in the images and determine area of the region inside of the boundary. Subsequently, the thickness of the developed precipitate region and the cross sectional area for flow for the inner and outer fluid were calculated. Results were verified manually from the grayscale cross-section images. The longitudinal (parallel to flow) cross sections were generated in ImageJ using the horizontal cross-section image stacks.

Experimental Design. All fluids were degassed prior to use by stirring under vacuum. A constant volumetric flow of $Q_{\text {inner }}+$ $Q_{\text {outer }}=390 \mathrm{~mL} / \mathrm{h}$ was used, and experiments were conducted under two relative flow rate conditions: 1) a 1:1 flow ratio with rates $Q_{\text {outer }}=Q_{\text {inner }}=195 \mathrm{~mL} / \mathrm{h}$, and 2) a 3:1 flow ratio, with the greater flow rate on the outside, $Q_{\text {outer }}=292 \mathrm{~mL} / \mathrm{h}, Q_{\text {inner }}=$ $98 \mathrm{~mL} / \mathrm{h}$. In each case, the flow was initiated using deionized water so that the flow profile was established when the sources were switched to the $\mathrm{Na}_{2} \mathrm{CO}_{3}$ and $\mathrm{CaCl}_{2}$ reactant solutions at the beginning of the experiments. From the Ergun equation for 


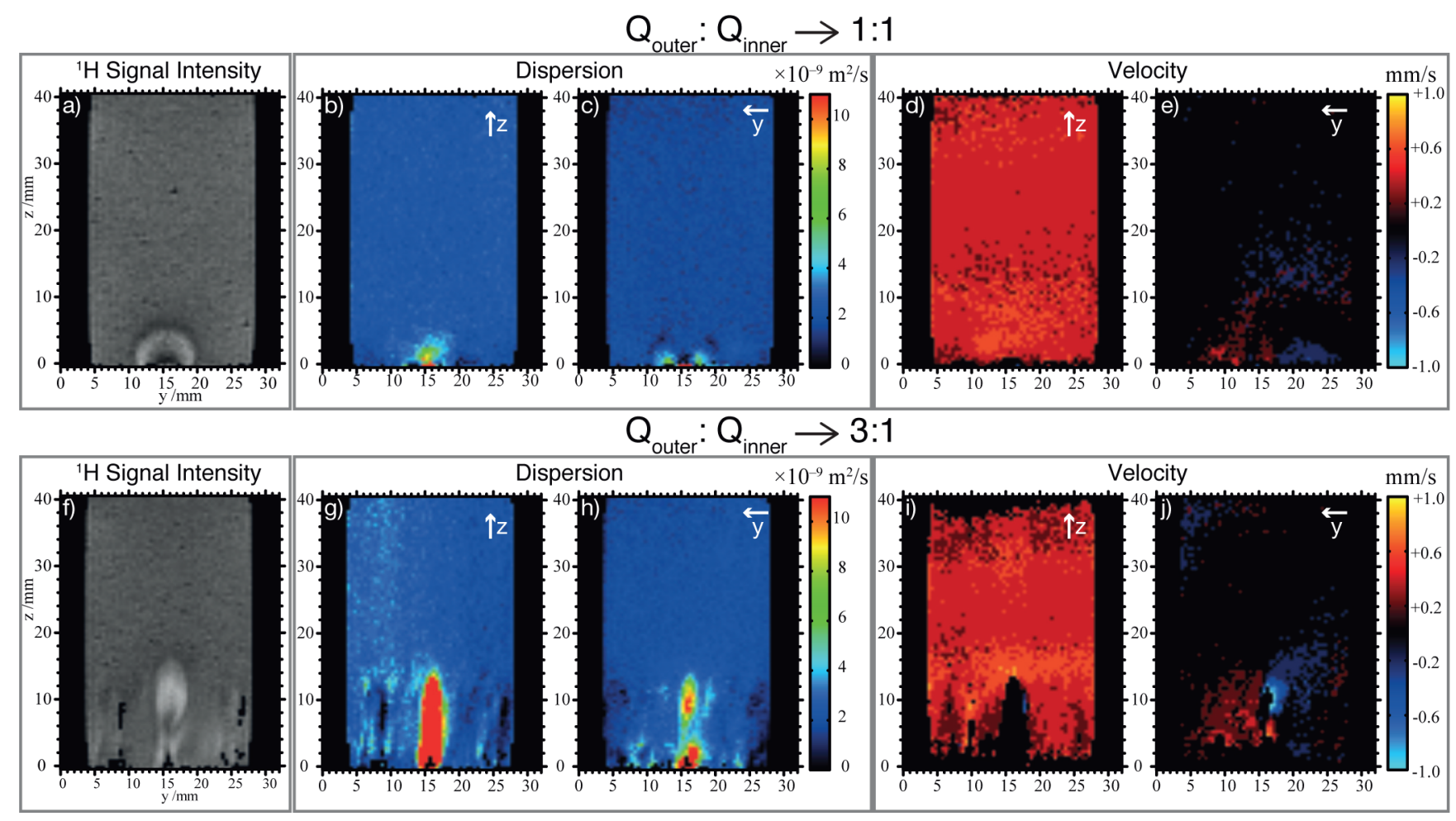

Figure 2. MRI ${ }^{1} \mathrm{H}$ signal intensity (in arbitrary units), dispersion, and velocity maps with deionized water injected in both inlets prior to precipitate formation. White arrows indicate the direction of motion sensitization. $(\mathbf{a}-\mathbf{e})$ The $Q_{\text {outer }}=Q_{\text {inner }}(1: 1)$ flow condition and $\left(f_{-j} j\right)$ the $Q_{\text {outer }}=3 \times Q_{\text {inner }}$ (3:1) flow condition.

incompressible flow, the ratio of volumetric flow rates $(Q)$ of the inner and outer flows are equal to the ratio of areas $(A)$ : $\frac{Q_{\text {outer }}}{Q_{\text {inner }}}=\frac{A_{\text {outer }}}{A_{\text {inner }}}=\frac{R_{\text {column }}^{2}-R_{\text {precip }}^{2}}{R_{\text {precip }}^{2}}$, where $R$ is radius of the column $\left(R_{\text {column }}\right)$ or of the zone inside the precipitate $\left(R_{\text {precip }}\right)$. The relative contributions of advection and diffusion to transport, which interact to generate hydrodynamic dispersion, is given by the Péclet number $P e=v l / D_{\text {eff }}$ where $l$ is a pore length scale based on grain size. Table 1 presents the Reynolds $(R e=\rho v l / \mu)$ and Péclet numbers for the different flow rate ratios at the point near the inlet where the two fluids first come in contact and in the region where the fluid velocity has stabilized.

An initial $\mu$-CT scan was conducted on the clean bead pack with water under no flow. MRI measurements were then conducted in three sequential system conditions: 1) no-flow, with water stationary in the bead pack; 2) flow with water in both lines; then 3) flow with the reactive $\mathrm{Na}_{2} \mathrm{CO}_{3} / \mathrm{CaCl}_{2}$ solutions. A final $\mu$-CT scan was performed after terminating flow to map the $\mathrm{CaCO}_{3}$ precipitate zone that had formed. Prior to transfer of the flow cell between the MRI and CT imaging systems, the cell was flushed with water saturated relative to $\mathrm{CaCO}_{3(\mathrm{~s})}$ to minimize the potential for further reactions (dissolution or precipitation). The saturated solution was produced by mixing the two $\mathrm{CaCl}_{2}$ and $\mathrm{Na}_{2} \mathrm{CO}_{3}$ solutions used in the experiments and allowing precipitation to occur under mixing for $24 \mathrm{~h}$, followed by filtration $(0.2 \mu \mathrm{m}$ pore size $)$ to remove the solid that had formed. Although the absence of dissolution or precipitation was not verified quantitatively, it is supported by the similarity between the final MR image and end point CT image in each experiment. After each experiment, the bead pack was removed, and the entire apparatus was acid washed and reassembled with clean beads.

\section{RESULTS AND DISCUSSION}

MRI Prior to Precipitation Reaction. The MRI images are acquired in a longitudinal slice (parallel to flow) through the center of the column (see Figure 1a). Figure 1 also shows the ${ }^{1} \mathrm{H}$ signal intensity map, hydrodynamic dispersion, and velocity (zero for no-flow) measured in the longitudinal $(z)$ direction. Maps of dispersion and velocity in the transverse $(y)$ direction are not shown but were identical, confirming that effective diffusion was isotropic on the scale of the $500 \mu \mathrm{m}$ image resolution and that velocity was zero in the transverse direction as well. The effective diffusion coefficient in both directions is $D_{\text {eff }} \sim(1.4 \pm 0.1) \times 10^{-9} \mathrm{~m}^{2} / \mathrm{s}$. Given the free diffusion of water is $D_{0} \sim(2.1 \pm 0.1) \times 10^{-9} \mathrm{~m}^{2} / \mathrm{s}$, our measured value is about $0.7 D_{0}$, which is consistent with the results in Mair et al. ${ }^{31}$ and Latour et al. ${ }^{32}$ where it has been shown that a value of $0.7 D_{0}$ occurs when the mean-square displacement of the molecules in a spherical bead pack is about $10 \%$ of the bead diameter $(20 \mu \mathrm{m}$ in our case). Our value is of the same order of magnitude reduction in $D(\Delta)$ as observed in previous work.

Figure 2 shows the spin-density, dispersion, and velocity maps for flow of water in the column prior to introduction of the reactant solutions. In the 1:1 flow condition a small entrance region extends from $z \sim 0 \mathrm{~mm}$ at the inlet to $z \sim 5$ $\mathrm{mm}$, while for the 3:1 flow condition a jet-like entrance region is clearly visible out to a distance $z \sim 15 \mathrm{~mm}$. Slight differences in column assembly (alignment of the inner inlet, packing efficiency, etc.) may be responsible for the extent of the inlet jet. An MRI in-flow artifact (discussed above) produces a bright spot in the ${ }^{1} \mathrm{H}$ signal intensity images near the inlet because of the rapid influx of water protons that have not yet reached NMR saturation. This bright spot does not represent an actual increase in spin density because, at the conclusion of the 


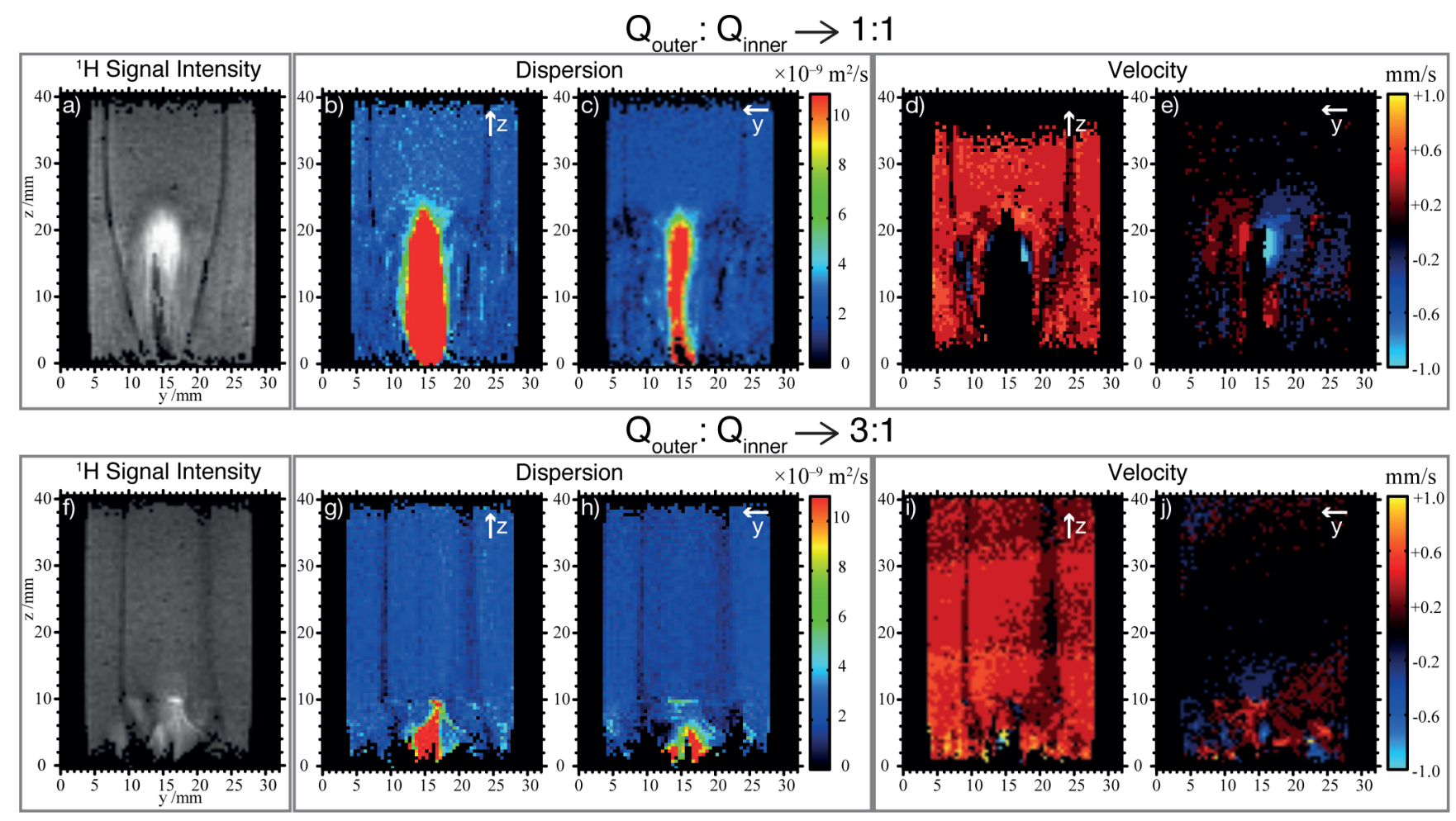

Figure 3. MRI ${ }^{1} \mathrm{H}$ signal intensity (in arbitrary units), dispersion, and velocity maps after $2-3 \mathrm{~h}$ of flow and precipitate formation. White arrows indicate the direction of motion sensitization. (a-e) The $Q_{\text {outer }}=Q_{\text {inner }}(1: 1)$ flow condition and $(\mathbf{f}-\mathbf{j})$ the $Q_{\text {outer }}=3 \times Q_{\text {inner }}(3: 1)$ flow condition.

experiment, it was observed that the tight cell packing had remained intact, and beads had not moved due to fluid shear force. Additionally, the rapidly fluctuating fluid motion near the inlet produces phase-encoding motion artifacts ${ }^{33}$ that limit accurate determination of dispersion and velocity in this region; consequently quantitative analysis of the MRI data is restricted to the region beyond the entrance length.

After entrance effects have diminished, at $z \geq 20 \mathrm{~mm}$, the longitudinal and transverse dispersion coefficients are $D_{\mathrm{L}} * \sim$ $(2.8 \pm 0.4) \times 10^{-9} \mathrm{~m}^{2} / \mathrm{s}$ and $D_{\mathrm{T}}{ }^{*} \sim(2.1 \pm 0.3) \times 10^{-9} \mathrm{~m}^{2} / \mathrm{s}$, in excellent agreement with dispersion values at $P e=31$ from a variety of measurements on consolidated granular porous media. ${ }^{27}$ The mapped velocity fields show that, for both the $1: 1$ and 3:1 inlet flow conditions, there is a uniform longitudinal and zero transverse velocity after a flow entrance length of $z \geq$ $20 \mathrm{~mm}$. The dispersion maps indicate uniform dispersivity in both longitudinal and transverse directions after the entrance length and prior to precipitation. Note that the velocity map of Figure $2 \mathrm{j}$ ) shows evidence of a recirculation zone near the jet in the region $y \sim 16 \mathrm{~mm}$ compared with the more symmetric flow seen in Figure 2e). The occurrence of a recirculation zone may be due to slight off-center positioning of the inner inlet tubing.

MRI during Precipitation. The impact of the precipitation reaction on the hydrodynamic dispersion and velocity fields is visualized in Figure 3. In the 1:1 flow rate case, the distance between the inlet and the establishment of a radially uniform dispersion and velocity field is $z \sim 25 \mathrm{~mm}$. Up to that point, the radial position of the precipitation reaction front expands with distance into the column (i.e., along the $z$ coordinate). At $z \sim$ $25 \mathrm{~mm}$ and beyond, the reaction front reaches an equilibrium radial position with $r_{\text {inner }} \sim 8.6 \mathrm{~mm}$ and $r_{\text {outer }} \sim 9.4 \mathrm{~mm}$. The effective diffusion coefficient in the precipitation zone (zero velocity), $D_{\text {eff }} \sim(1.2 \pm 0.3) \times 10^{-9} \mathrm{~m}^{2} / \mathrm{s}$, is reduced relative to the effective diffusion coefficient in the no-flow bead pack prior to precipitation. The longitudinal and transverse velocities in the region of precipitation are reduced to near zero indicating the permeability is significantly decreased. ${ }^{34}$ This suggests that the primary mechanism for transport, if any, within the precipitation zone is molecular diffusion. Given the significant reduction of porosity by the precipitate, the system may be in a regime where the local diffusion measurement is scaled by the tortuosity, ${ }^{31} D_{\text {eff }} \sim D_{0} / \tau$. While the tortuosity in the $\mathrm{CaCO}_{3}$ layer is not known, predicted values for saturated porous media at similar porosities range between $1.5-3.0$, yielding $D_{\text {eff }}$ values consistent with our measurements. $^{32,35}$ In the $3: 1$ case, there is a difference in thickness of the precipitation zone on opposite sides of the cell, and the dispersion and velocity of the fluid become uniform over a shorter distance of about $10 \mathrm{~mm}$. The measured effective diffusion and velocity in the precipitation zone are similar to the $1: 1$ case.

The noninvasive nature of the MRI measurement permits observation of the precipitation zone as it forms. The spindensity images and their associated dispersion maps in SI Figure $\mathrm{S} 1$ depict the formation of a precipitate region over successive $30 \mathrm{~min}$ image acquisition cycles for the 1:1 flow case. A steady state appears to be established after 60-90 min and persists over the remainder of the experiment time $(180 \mathrm{~min})$. The same image sequence for the 3:1 flow case is shown in SI Figure S2.

$\boldsymbol{\mu}$-CT Imaging. Micro-CT scans taken before each flow experiment indicated a clean, homogeneously distributed bead pack prior to addition of the reacting solutions. After both flow experiments, the $\mu$-CT images indicated a region of precipitation had formed where a bright (dense) region was visible in the column between the approximate areas of flow for the inner and outer fluids (Figure $4 \mathrm{~b}, \mathrm{e}$ ). Although the boundary of the precipitate was apparent in the images, the resolution was not sufficient to determine the porosity. Stark 


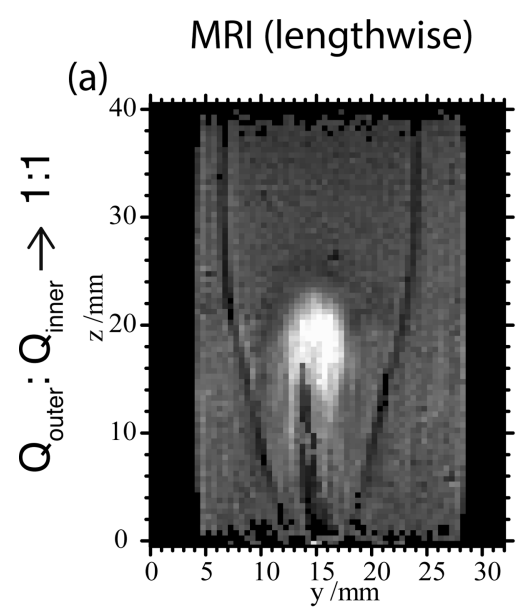

(d)

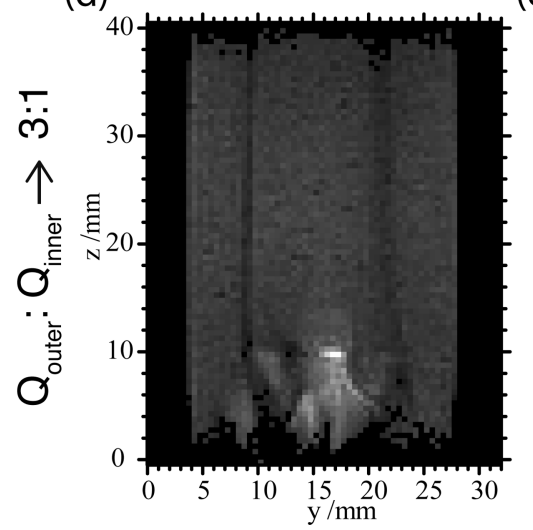

(b)

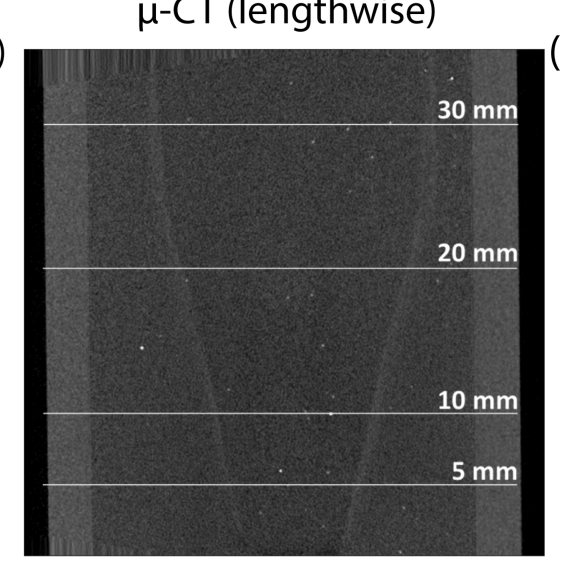

(e)

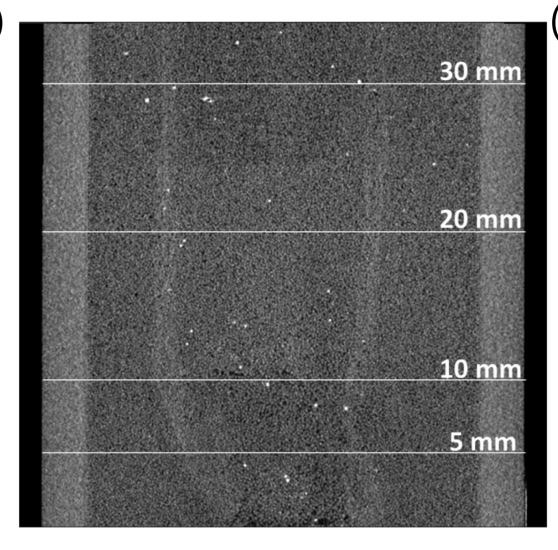

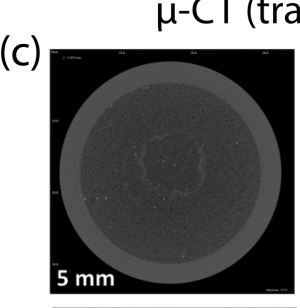
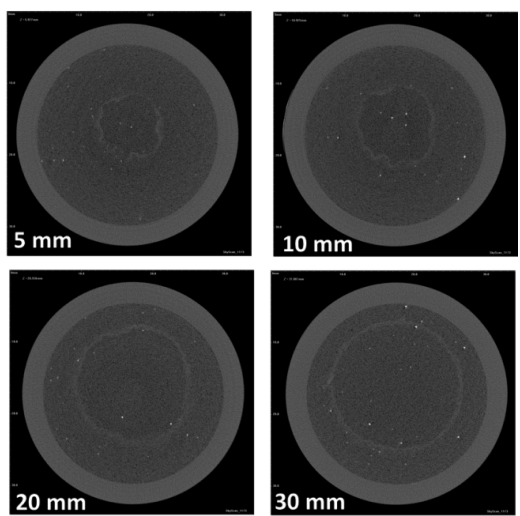

$(f)$
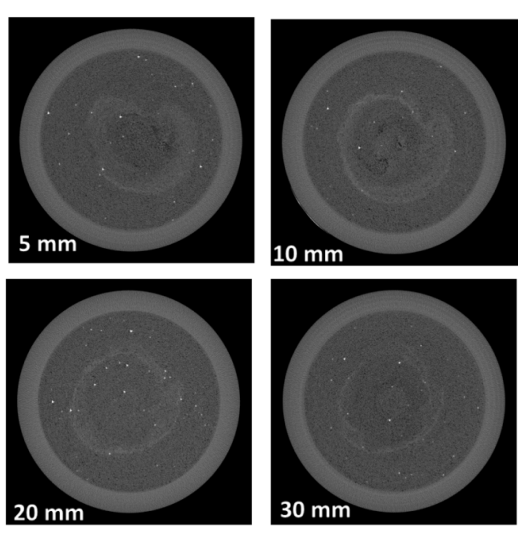

Figure 4. Comparison of MRI and $\mu$-CT images for (a-c) the $Q_{\text {outer }}=Q_{\text {inner }}(1: 1)$ flow condition and (d-f) the $Q_{\text {outer }}=3 \times Q_{\text {inner }}(3: 1)$ flow condition. The MRI ${ }^{1} \mathrm{H}$ signal intensity images $(\mathbf{a}, \mathbf{d})$ were acquired during flow, and the precipitate appears as a reduction in signal intensity (darker voxels). The coplanar X-ray $\mu$-CT images (b, e) were acquired following the flow experiment and show precipitate as an increase in signal intensity (brighter voxels). The position of inlet $\mathrm{I}(\mathrm{z}=0)$ is at the bottom edge of the images. The white lines on the lengthwise $\mu$-CT images indicate the position of corresponding $\mu$-CT horizontal cross-section images (c, $\mathrm{f}$ ).

differences in the geometry, thickness, and morphology of the precipitate zone in the inlet region between the two conditions were however observed and correlated to results obtained by the real-time MRI.

Comparison of MRI and $\mu$-CT Results. MRI and $\mu$-CT images (Figure 4) were compared to establish correlations between the spatial distribution of precipitates and the signals obtained by the two imaging methods. In both flow rate cases, the shape of the precipitation zone coincided for the MRI and $\mu$-CT images.

In both flow rate conditions, an entrance region was observed near the inlets that extended into the cell over the distance required for the inner and outer flows to reach equal velocity. In the 1:1 flow condition, the calculated $R e$, and thus the fluid velocity, for the $\mathrm{Na}_{2} \mathrm{CO}_{3}$ solution at the inner column inlet is higher than the $\mathrm{CaCl}_{2}$ solution at the outer inlet (Table 1 ), thus the cross-sectional area available for flow of the $\mathrm{Na}_{2} \mathrm{CO}_{3}$ solution increased in the direction of flow to create the shape of the precipitate cone observed in Figure $4 a$ and $b$. In the region in which the radial precipitate zone position is changing, longitudinal and transverse dispersions are greater in the inner region, and transverse velocity components are present (Figure 3). In Figure 4, $\mu$-CT cross sectional images for the $1: 1$ flow rates of reactants show the precipitation zone at $z$ $\sim 5 \mathrm{~mm}$ and $10 \mathrm{~mm}$ from the central inlet has a wavy spatial pattern, which becomes more regular as the transverse velocity subsides and the transverse and longitudinal dispersion is homogenized. At $z \geq 30 \mathrm{~mm}$, where radial position of the precipitate zone is no longer changing along the direction of flow, the zone is thinner and has a more regular circular spatial distribution compared to the wavy pattern in the entrance region. The hydrodynamic instabilities associated with the entrance flow effects, as quantified by the enhanced velocity fluctuations and hydrodynamic dispersion in this region, affect the structure of the reaction front. This suggests a strong analogy to the surface reaction flow models in which velocity fluctuation intensity induces differing precipitate structure formation. ${ }^{36}$ However, once the precipitation zone is established, it is stable and reduces the role of flow variations, or turbulence, on reactant mixing.

Relative to the $1: 1$ flow condition, the distance required to establish a uniform cylindrical precipitation zone in the 3:1 flow condition (lower inner flow rate) is shorter. The precipitate deposition zone attains a maximum radius at $z \sim 10 \mathrm{~mm}$ as shown in the $\mu$-CT and MRI scans (Figure 4d, e). In this case, $R e$ and velocity for the two fluids in the inlet region of the column are closer in value (see Table 1), and a shorter distance is required to reach the equilibrated flow condition. The precipitate reaction front formed is thicker, the density and shape of the precipitate appears less homogeneous, and the circular spatial distribution is less regular at distances 20-30 $\mathrm{mm}$ from the inlet (Figure $4 \mathrm{f}$ ). A notable feature is the 
asymmetric "bulge" in the precipitate region near the inlet region at $z \sim 5-10 \mathrm{~mm}$, visible in both the MRI and $\mu$-CT images. This feature corresponds to the recirculation instability seen in the flow velocities in the unreacted bead pack (Figure $2 \mathrm{j}$ ), and it is possible that the overall greater thickness and asymmetry of the precipitate zone in the 3:1 flow case may be due to enhanced velocity fluctuations and dispersion associated with this hydrodynamic instability.

Analysis of the horizontal cross-sectional $\mu$-CT images of Figure $4 \mathrm{c}$ indicates that for 1:1 volumetric reactant flow rates the cross sectional flow areas at lengths $z \geq 30 \mathrm{~mm}$ are $A_{\text {inner }} \sim$ $232 \mathrm{~mm}^{2}$ and $A_{\text {outer }} \sim 224 \mathrm{~mm}^{2}$ and are separated by the observable precipitate zone. As stated above, the $\mu$-CT image analysis for the 1:1 flow condition resulted in an average precipitation zone thickness of $0.8 \pm 0.1 \mathrm{~mm}$ at a distance of 30 $\mathrm{mm}$ from the inlet. This agrees with the precipitation zone thickness of $0.8 \mathrm{~mm}$ determined from the MRI data (Figure 3). In contrast, the 3:1 flow rates produced cross sectional areas for flow of $A_{\text {inner }} \sim 93 \mathrm{~mm}^{2}$ and $A_{\text {outer }} \sim 309 \mathrm{~mm}^{2}$ at $z=20 \mathrm{~mm}$ from the inlet (Figure $4 \mathrm{f}$ ), reflecting the ratios of the inner to outer flow rates. These cross sectional areas for inner and outer fluid flows result in an average precipitate thickness of $2.4 \pm 0.5$ $\mathrm{mm}$, which is a factor of 3 greater than the precipitate thickness for the 1:1 equal flow rate experiment.

These results show that the relative reactant flow rates and the resulting pore scale hydrodynamics control the location of the precipitation front and may impact the structure and properties of the mixing/precipitation zone. The precipitate front between the two fluids in the equilibrated region resulted in the radial position of the precipitation layer predicted by the Ergun equation (see the Experimental Design section) for flow of each solution of $R_{\text {precip }}=R_{\text {column }} / 2^{1 / 2}=8.84 \mathrm{~mm} \sim 8.6 \pm 0.8$ $\mathrm{mm}$ for $\frac{Q_{\text {outer }}}{Q_{\text {inner }}}=1:$ land $R_{\text {precip }}=R_{\text {column }} / 2=6.25 \mathrm{~mm} \sim 5.5 \pm$ $2.4 \mathrm{~mm}$ for $\frac{Q_{\text {outer }}}{Q_{\text {inner }}}=3: 1$. The thickness of the precipitate region was inversely related to the degree of dispersive mixing and was visualized directly with $\mu$-CT.

The complementary image data obtained via MRI and $\mu$-CT uniquely permit investigation of the coevolution of flow, mixing, and changes in media structure due to the formation of precipitates in the porous medium. Clear and significant reductions in dispersion and velocity in the precipitate region were observed, confirming that porosity was reduced. This indicates that different relative reactant flow rates can potentially be used to control the curvature, density, and steady-state position of precipitate layers in porous media.

Importantly, coupling the measurement of fluid transport, measured by MRI, and the precipitate structure, measured by MRI and $\mu$-CT, indicates that the thickness, spatial location, and structure of the precipitate layer prior to the steady-state region is determined by the enhanced dispersion and velocity fluctuations generated by the observed hydrodynamic instabilities. These instabilities can be quantified in the MRI velocity data as secondary, nonaxial flows. The presence of hydrodynamic instabilities will vary significantly in $2 \mathrm{D}$ relative to $3 \mathrm{D}$ and demonstrate the importance of $3 \mathrm{D}$ investigations of precipitate formation in porous media and the value of an experimental approach that eliminates possible artifacts due to wall effects in the flow cell. These results suggest that the injection rate of aqueous reactants may be used to engineer specific structures or morphologies during the establishment of $\mathrm{CaCO}_{3}$ or other mineral precipitate barriers.
Future investigations using these methods may focus on applying combinations of MRI and $\mu$-CT imaging in experiments conducted under relevant environmental conditions (e.g., with respect to pressure, temperature, and solution chemistry). For example, representative geologic material, either consolidated or unconsolidated, can be inserted into MRI and X-ray compatible flowcells ${ }^{37}$ (such as Hassler-type coreholders made of PEEK or other compatible materials). Xray $\mu$-CT can be used for high resolution imaging of the pore space, while MRI will allow for observation of flow dynamics as well as imaging (at slightly lower resolution than $\mu$-CT). The combination of both techniques is expected to provide significant insight into microscale reaction and transport processes in porous media as well as their influence on porosity, permeability, flow (path) dynamics, solute mixing, and volume-averaged reaction rates.

\section{ACKNOWLEDGMENTS}

A portion of this research was conducted under DOE Idaho Operations Office Contract DE-AC07-05ID14517, with funding provided by the U.S. Department of Energy (DOE), Office of Science, Subsurface Biogeochemical Research (SBR) Program. J.D.S. and S.L.C. acknowledge the U.S. NSF Major Research Instrumentation program and the M.J. Murdock Charitable Trust for MR equipment and $\mu$-CT instrument funding. R.G. and E.L. acknowledge the U.S. DOE, Office of Science, SBR Program, contract no. DE-FG-02-09ER64758; additional support was provided through grant numbers DEFE0004478, DE-FE0009599, and DE-FG02-13ER86571 and by the National Science Foundation's Collaborations in Mathematical Geosciences (CMG) program award no. DMS0934696.

\section{REFERENCES}

(1) Fogler, H. S. Elements of Chemical Reaction Engineering. Prentice Hall: Englewood Cliffs, NJ, 1986.

(2) Bedient, P. B.; Rifai, H. S.; Newell, C. J. Ground Water Contamination: Transport and Remediation; PRT Prentice-Hall, Inc.: NJ, 1994.

(3) Fujita, Y.; Taylor, J. L.; Gresham, T. L. T.; Delwiche, M. E.; Colwell, F. S.; McLing, T. L.; Petzke, L. M.; Smith, R. W. Stimulation of microbial urea hydrolysis in groundwater to enhance calcite precipitation. Environ. Sci. Technol. 2008, 42 (8), 3025-3032. 
(4) Wu, Y. X.; Ajo-Franklin, J. B.; Spycher, N.; Hubbard, S. S.; Zhang, G. X.; Williams, K. H.; Taylor, J.; Fujita, Y.; Smith, R. Geophysical monitoring and reactive transport modeling of ureolytically-driven calcium carbonate precipitation. Geochem. Trans. 2011, 12 (1), 7.

(5) Connolly, J. M.; Gerlach, R. Microbially induced carbonate precipitation in the subsurface: Fundamental reaction and transport processes. In Handbook of Porous Media, 3rd ed.; Vafai, K., Ed.; CRC Press: 2015.

(6) Emmanuel, S.; Berkowitz, B. Mixing-induced precipitation and porosity evolution in porous media. Adv. Water Resour. 2005, 28 (4), 337-344.

(7) Singh, R.; Yoon, H.; Sanford, R. A.; Katz, L.; Fouke, B. W.; Werth, C. J. Metabolism-Induced CaCO3 Biomineralization During Reactive Transport in a Micromodel: Implications for Porosity Alteration. Environ. Sci. Technol. 2015, 49 (20), 12094-12104.

(8) Phillips, A. J.; Gerlach, R.; Lauchnor, E.; Mitchell, A. C.; Cunningham, A. B.; Spangler, L. Engineered applications of ureolytic biomineralization: a review. Biofouling 2013, 29 (6), 715-33.

(9) Singurindy, O.; Berkowitz, B.; Lowell, R. P. Carbonate dissolution and precipitation in coastal environments: Laboratory analysis and theoretical consideration. Water Resour. Res. 2004, 40, W04401.

(10) Dentz, M.; Le Borgne, T.; Englert, A.; Bijeljic, B. Mixing, spreading and reaction in heterogeneous media: A brief review. $J$. Contam. Hydrol. 2011, 120-121, 1-17.

(11) Zhang, C.; Dehoff, K.; Hess, N.; Oostrom, M.; Wietsma, T. W.; Valocchi, A. J.; Fouke, B. W.; Werth, C. J. Pore-Scale Study of Transverse Mixing Induced $\mathrm{CaCO} 3$ Precipitation and Permeability Reduction in a Model Subsurface Sedimentary System. Environ. Sci. Technol. 2010, 44 (20), 7833-7838.

(12) Lauchnor, E. G.; Schultz, L. N.; Bugni, S.; Mitchell, A. C.; Cunningham, A. B.; Gerlach, R. Bacterially Induced Calcium Carbonate Precipitation and Strontium Coprecipitation in a Porous Media Flow System. Environ. Sci. Technol. 2013, 47 (3), 1557-1564.

(13) Tartakovsky, A. M.; Redden, G.; Lichtner, P. C.; Scheibe, T. D.; Meakin, P. Mixing-induced precipitation: Experimental study and multiscale numerical analysis. Water Resour. Res. 2008, 44, W06S04.

(14) Yoon, H.; Valocchi, A. J.; Werth, C. J.; Dewers, T. Pore-scale simulation of mixing-induced calcium carbonate precipitation and dissolution in a microfluidic pore network. Water Resour. Res. 2012, 48, W02524.

(15) Callaghan, P. T. Translational Dynamics and Magnetic Resonance: Principles of Pulsed Gradient Spin Echo NMR; Oxford University Press: 2011; p 568.

(16) Seymour, J. D.; Callaghan, P. T. Generalized approach to NMR analysis of flow and dispersion in porous media. AIChE J. 1997, 43 (8), 2096-2111.

(17) Fridjonsson, E. O.; Seymour, J. D.; Schultz, L. N.; Gerlach, R.; Cunningham, A. B.; Codd, S. L. NMR measurement of hydrodynamic dispersion in porous media subject to biofilm mediated precipitation reactions. J. Contam. Hydrol. 2011, 120-121, 79-88.

(18) Sham, E.; Mantle, M. D.; Mitchell, J.; Tobler, D. J.; Phoenix, V. R.; Johns, M. L. Monitoring bacterially induced calcite precipitation in porous media using magnetic resonance imaging and flow measurements. J. Contam. Hydrol. 2013, 152, 35-43.

(19) Handley-Sidhu, S.; Sham, E.; Cuthbert, M. O.; Nougarol, S.; Mantle, M.; Johns, M. L.; Macaskie, L. E.; Renshaw, J. C. Kinetics of urease mediated calcite precipitation and permeability reduction of porous media evidenced by magnetic resonance imaging. Int. J. Environ. Sci. Technol. 2013, 10 (5), 881-890.

(20) Wildenschild, D.; Sheppard, A. X-ray imaging and analysis techniques for quantifying pore-scale structure and processes in subsurface porous medium systems. Adv. Water Resour. 2013, 51, 217-246.

(21) Cnudde, V.; Masschaele, B.; Dierick, M.; Vlassenbroeck, J.; Van Hoorebeke, L.; Jacobs, P. Recent progress in X-ray CT as a geosciences tool. Appl. Geochem. 2006, 21 (5), 826-832.
(22) Armstrong, R.; Ajo-Franklin, J. Investigating biomineralization using synchrotron based X-ray computed microtomography. Geophy Res. Lett. 2011, 38, L08406.

(23) von der Schulenburg, D. A. G.; Paterson-Beedle, M.; Macaskie, L. E.; Gladden, L. F.; Johns, M. L. Flow through an evolving porous media-compressed foam. J. Mater. Sci. 2007, 42 (16), 6541-6548.

(24) Dohnal, M.; Jelinkova, V.; Snehota, M.; Dusek, J.; Brezina, J. Tree-Dimensional Numerical Analysis of Water Flow Affected by Entrapped Air: Application of Noninvasive Imaging Techniques. Vadose Zone J. 2013, 12 (1), 1-12.

(25) Votrubová, J.; Císlerová, M.; Gao Amin, M. H.; Hall, L. D. Recurrent ponded infiltration into structured soil: A magnetic resonance imaging study. Water Resour. Res. 2003, 39 (12), 1371.

(26) Rigby, S. P.; Watt-Smith, M. J.; Chigada, P.; Chudek, J. A.; Fletcher, R. S.; Wood, J.; Bakalis, S.; Miri, T. Studies of the entrapment of non-wetting fluid within nanoporous media using a synergistic combination of MRI and micro-computed X-ray tomography. Chem. Eng. Sci. 2006, 61 (23), 7579-7592.

(27) Seymour, J. D.; Callaghan, P. T. Generalized approach to NMR analysis of flow and dispersion in porous medium. AIChE J. 1997, 43, 2096-2111.

(28) Feldkamp, L.; Davis, L.; Kress, J. Practical cone-beam algorithm. J. Opt. Soc. Am. A 1984, 1 (6), 612-619.

(29) Schneider, C.; Rasband, W.; Eliceiri, K. NIH Image to ImageJ: 25 years of image analysis. Nat. Methods 2012, 9 (7), 671-675.

(30) Otsu, N. Threshold selection method from gray-level histograms. IEEE Trans Syst. Man Cybern Syst 1979, 9 (1), 62-66.

(31) Mair, R. W.; Wong, G. P.; Hoffmann, D.; Hürlimann, M. D.; Patz, S.; Schwartz, L. M.; Walsworth, R. L. Probing Porous Media with Gas Diffusion NMR. Phys. Rev. Lett. 1999, 83 (16), 3324-3327.

(32) Latour, L. L.; Mitra, P. P.; Kleinberg, R. L.; Sotak, C. H. TimeDependent Diffusion Coefficient of Fluids in Porous Media as a Probe of Surface-to-Volume Ratio. J. Magn. Reson., Ser. A 1993, 101 (3), $342-346$.

(33) Callaghan, P. T. Principles of Nuclear Magnetic Resonance Microscopy; Oxford University Press: New York, 1991.

(34) Seto, K.; Hollenshead, J. T.; Watson, A. T.; Chang, C. T. P.; Slattery, J. C. Determination of permeability distributions using NMR velocity imaging. Transp. Porous Media 2001, 42, 351-388.

(35) Ghanbarian, B.; Hunt, A. G.; Ewing, R. P.; Sahimi, M. Tortuosity in Porous Media: A Critical Review. Soil Sci. Soc. Am. J. 2013, 77 (5), 1461-1477.

(36) Goldenfeld, N.; Chan, P. Y.; Veysey, J. Dynamics of Precipitation Pattern Formation at Geothermal Hot Springs. Phys. Rev. Lett. 2006, 96 (25), 254501.

(37) Prather, C. A.; Bray, J. M.; Seymour, J. D.; Codd, S. L. NMR study comparing capillary trapping in Berea sandstone of air, carbon dioxide, and supercritical carbon dioxide after imbibition of water. Water Resour. Res. 2016, 52 (2), 713-724. 